\title{
Development of positron annihilation spectroscopy for investigating deuterium decorated voids in neutron-irradiated tungsten
}

\author{
C.N. Taylor ${ }^{\mathrm{a}^{*}}$, M. Shimada ${ }^{\mathrm{a}}$, B.J. Merrilla, D.W. Akers ${ }^{\mathrm{b}}$, Y. Hatano ${ }^{\mathrm{c}}$ \\ ${ }^{a}$ Fusion Safety Program, Idaho National Laboratory, Idaho Falls, ID 83415, USA \\ ${ }^{b}$ Experimental Programs, Idaho National Laboratory, Idaho Falls, ID 83415, USA \\ ${ }^{c}$ Hydrogen Isotope Research Center, University of Toyama, Toyama 930-8555, Japan
}

\section{Abstract (150 words max)}

The present work is a continuation of a recent research to develop and optimize positron annihilation spectroscopy (PAS) for characterizing neutron-irradiated tungsten. Tungsten samples were exposed to neutrons in the High Flux Isotope Reactor (HFIR) at Oak Ridge National Laboratory and damaged to 0.025 and $0.3 \mathrm{dpa}$. Subsequently, they were exposed to deuterium plasmas in the Tritium Plasma Experiment (TPE) at Idaho National Laboratory. The implanted deuterium was desorbed through sample heating to $900^{\circ} \mathrm{C}$, and Doppler broadening (DB)-PAS was performed both before and after heating. Results show that deuterium impregnated tungsten is identified as having a smaller S-parameter. The Sparameter increases after deuterium desorption. Microstructural changes also occur during sample heating. These effects can be isolated from deuterium desorption by comparing the S-parameters from the deuterium-free back face with the deuterium-implanted front face. The application of using DB-PAS to examine deuterium retention in tungsten is examined.

PACS: 78.70.Bj (positron annilation), 61.80.Hg (neutron damage), 28.52.Cx (fusion safety), 52.77.Dq (PMI-ion implantation/desorption).

PSI-21 keywords: Tungsten, Deuterium, Retention, Neutron damage, Doppler broadening

"Corresponding author address: P.O. Box 1625, Idaho Falls, ID 83415-7113

*Corresponding author E-mail: chase.taylor@inl.gov

Presenting author: C.N. Taylor

Presenting author E-mail: chase.taylor@inl.gov 


\section{Introduction}

Neutron and ion damage to plasma-facing components (PFC) in fusion reactors will result in the production of voids and bubbles, which act as trapping sites for deuterium and tritium [1]. Tritium retention poses concerns for fuel management and accident scenarios. Neutron irradiation creates damage throughout the bulk of samples and deuterium retention differs compared to tungsten damage produce via ion beams [2,3]. Measuring retention usually is achieved through thermal desorption spectroscopy (TDS) or nuclear reaction analysis (NRA). Both techniques have valuable properties yet drawbacks. High temperatures used in TDS ensure full deuterium desorption but can alter the microstructure of the sample due to defect recovery, limiting parallel interrogation. The depth resolution of NRA is limited, and the high-energy ion beam used in the analysis may produce undesired changes in the sample. Positron annihilation spectroscopy (PAS) can be used as a non-destructive technique to assess the microstructural changes to the sample, and in special applications, it can be used to obtain information regarding the chemical state of the material [4]. The work in this paper builds off of previous results [5], where the primary purpose of the present work is to examine the before and after effects of TDS using Doppler broadening PAS (DB-PAS). We will show that both deuterium desorption and microstructural recovery affect the S-parameter during TDS, and provide suggestions for deconvoluting the two phenomena.

\section{Experimental details}

Samples were fabricated from 99.99 at.\% purity tungsten (A.L.M.T. Corp.) and cut from a $6 \mathrm{~mm}$ diameter rod to be $0.2 \mathrm{~mm}$ thick with the grains perpendicular to the sample face. The surface was mechanically polished and the samples were annealed to $900^{\circ} \mathrm{C}$ for 30 minutes prior to neutron-irradiation. As part of the 2007-2012 US/Japan Tritium, Irradiation and Thermofluids for America and Nippon (TITAN) project, the samples used in this study were irradiated in the High Flux Isotope Reactor at Oak Ridge National Laboratory for 391 
hr. to achieve a displacements per atom (dpa) damage level of 0.3. The dpa calculation followed ASTM E693-12, with a conversion factor for tungsten [6,7]. Sample temperature during neutron irradiation was at coolant temperature $\left(50-70^{\circ} \mathrm{C}\right)$ and no thermal neutron spectrum filtering was used. Samples were allowed to decay $\sim 2$ years prior to shipping to Idaho National Laboratory (INL). The samples described presently were also used and discussed in a previous study [5]. One 0.3 dpa sample (ID: Y111) was conserved as a control sample and was not subjected to any further modification.

At INL, $100 \mathrm{eV}$ deuterium was implanted $\left(\Gamma=5 \times 10^{21} \mathrm{~m}^{-2} \mathrm{~s}^{-1}\right)$ into the two samples at $200^{\circ} \mathrm{C}$ (sample $\mathrm{Y} 107$ ) and $500^{\circ} \mathrm{C}$ (sample Y112) to a fluence of $5 \times 10^{25} \mathrm{~m}^{-2}$ in the Tritium Plasma Experiment (TPE). Tritium was not used for these samples in order to reduce contamination to allow shipment of samples to off-site facilities. Following the first implantation, samples were shipped to University of Wisconsin-Madison for nuclear reaction analysis (NRA). Deuterium concentration was measured using a $3.5 \mathrm{MeV}{ }^{3} \mathrm{He}$ beam via the $\mathrm{D}\left({ }^{3} \mathrm{He}, \mathrm{p}\right)^{4} \mathrm{He}$ nuclear reaction, and SIMNRA software was used to analyze the results [8]. Following the first NRA, samples were returned to INL, and a second series of implantation and NRA were repeated [9]. The time delay between the two TPE exposures was 30-60 days. The cumulative deuterium ion fluence was $1 \times 10^{26} \mathrm{~m}^{-2}$.

DB-PAS was performed at INL approximately 440 and 150 days following the second NRA for 0.025 dpa and 0.3 dpa samples, respectively. The PAS technique has been reviewed in detail elsewhere [10,11], and parameters relevant to this work are contained in Ref [5]. In short, unmoderated positrons from a source enter the sample and thermalize within about 10 ps. The positron scatters through the sample and may encounter a void. Voids tend to trap positrons as the positive ionic cores of the void surface repel positrons. The positrons eventually annihilate in the bulk or a void to produce two $511 \mathrm{keV}$ gamma rays, one of which is detected with a high purity germanium (HPGe) detector. Trapped positrons are more likely 
to annihilate with valence electrons, whose momentum is slightly less than that of core shell electrons. This distribution in gamma momentum results in Doppler broadening of the 511 $\mathrm{keV}$ annihilation peak. The primary metric for comparing DB-PAS results from one sample to another is the S-parameter; here the S-parameter is defined as the ratio of the counts in the center channels $(511 \pm 0.9 \mathrm{keV})$ divided by the total counts in the region of interest $(511 \pm 10$ $\mathrm{keV})$.

The PAS systems utilized Ortec GEM20P4 hyperpure germanium detectors with an efficiency of about $15 \%$ relative to a 3" x 3 " $\mathrm{NaI}(\mathrm{Tl})$ detector. The resolution at the ${ }^{60} \mathrm{Co} 1.33$ $\mathrm{MeV}$ line is about $1.7 \mathrm{keV}$. Two positron sources were used to interrogate different regions of the sample. Approximately $90 \%$ of positrons from ${ }^{22} \mathrm{Na}\left(\mathrm{t}_{1} / 2=2.6 \mathrm{y}, \mathrm{E}_{\max }=0.545 \mathrm{MeV}\right.$, $\left.\mathrm{E}_{\text {mean }}=0.26 \mathrm{MeV}\right)$ annihilate within $58 \mu \mathrm{m}$ of the sample surface, and $18 \%$ of ${ }^{68} \mathrm{Ge}\left(\mathrm{t}_{1 / 2}=275\right.$ $\mathrm{d}, \mathrm{E}_{\max }=1.88 \mathrm{MeV}, \mathrm{E}_{\text {mean }}=0.41 \mathrm{MeV}$ ) positrons pass through the $200 \mu \mathrm{m}$ tungsten samples without annihilating (based on models by Mourino et al. [12]). Therefore, these two unmoderated positron sources provide a near-surface and bulk evaluation of the neutron damaged samples. Additional details of the DB-PAS analysis for these experiments have been described elsewhere [5].

Thermal desorption spectroscopy (TDS) was performed at INL to measure deuterium retention [9]. Samples were heated with a ramp rate of $10{ }^{\circ} \mathrm{C} / \mathrm{min}$ to $900{ }^{\circ} \mathrm{C}$ and held for 30 minutes. The TDS system was calibrated with 3 deuterium leaks and a TDS run with no sample was used as a baseline reference for subsequent TDS runs.

PAS was repeated after TDS to assess the effect of desorption on the S-parameter. Due to equipment failure, a second DB-PAS systems was used to examine sample Y107 and Y112 following TDS. The S-parameter is affected by system geometry and as a consequence, different detector setups yield different S-parameters when analyzing the same sample. This effect can be corrected by normalizing the data to a control sample. The pre-TDS and post- 
TDS data were normalized to the S-parameter of sample Y111, which did not undergo any modification between scans with the two systems. Normalization in this manner introduces an error of $\sim 0.0019$ (arb.), which was determined by comparing the difference between Sparameters from the first DB-PAS system to the second DB-PAS system after normalization to Y111. Systematic uncertainty additionally introduces an error of 0.0007 (arb.). The combined error in S-parameters is 0.0020 (arb.).

\section{Results and discussion}

Figure 1 compares the DB-PAS results from two 0.3 dpa samples before and after TDS. The left-hand panes $(\mathrm{a}, \mathrm{c})$ represent the front face of the sample, and the right-hand panes $(\mathrm{b}$, d) show the S-parameter from the back face of the sample, where $90 \%$ of positrons from Na22 annihilate within $\sim 58 \mu \mathrm{m}$ of the surface and Ge-68 provides a more integrated measurement of the whole sample. The reference 0.3 dpa sample (triangle) did not undergo TDS but was included as a baseline between the two scans. The pre-TDS data were first reported in Ref. [5]. Considering the front deuterium implanted surface in Figure 1(a), the Sparameter for Y107 (D-implantation at $200^{\circ} \mathrm{C}$ ) after TDS is substantially higher than its preTDS counterpart. Impurity decorated voids typically have a lower S-parameter than empty voids $[5,13,14]$. This partially explains why the S-parameter increases after TDS for sample Y107. Desorption of deuterium, however, fails to explain why sample Y112 (D-implantation at $500^{\circ} \mathrm{C}$ ) in (a) has approximately the same S-parameter before and after TDS.

These differences are likely due to at least two competing phenomena that occur in the tungsten samples during TDS: deuterium desorption and microstructural recovery. First, an empty void yields a higher S-parameter than an decorated void; thus heating to $900^{\circ} \mathrm{C}$, which eliminates the implanted deuterium, increases the S-parameter. Secondly, the heating sequence initiates microstructural defect recovery, which also has two competing sub-

phenomena: i) decreasing the number density of voids (observed as a decrease in S- 
parameter), ii) increasing the defect size (increase the S-parameter). According to $\mathrm{H}$. Schueltz, stage III recovery begins at 350-400 C [15]. Samples Y107 and Y112 had deuterium implanted at $200^{\circ} \mathrm{C}$ and $500^{\circ} \mathrm{C}$, respectively. In Figure 1 (a) The right-hand most sample was heated to $500^{\circ} \mathrm{C}$ during deuterium implantation. At this temperature the neutroninduced monovacancies migrate and annihilate with self interstitials and clusters. The implantation temperature of $200^{\circ} \mathrm{C}$ for sample Y107 would not have been sufficient for stage III recovery to commence. Reducing the number of voids can lower the S-parameter, but if the size of the voids concurrently increases, the S-parameter may be unaffected.

The quantity of retained deuterium was also considered. The TDS and NRA results from these two samples are summarized in Figure 2 (analysis of TDS and NRA data will be detailed in an upcoming publication [9]). TDS measures deuterium desorbed from the entire sample bulk, whereas NRA detects deuterium within $\sim 6 \mu \mathrm{m}$ of the surface. The combined TDS and NRA results suggest that sample $\mathrm{Y} 112\left(500^{\circ} \mathrm{C}\right)$ retained more deuterium in the bulk than the sample Y107 $\left(200^{\circ} \mathrm{C}\right)$. Deuterium permeates deep into the bulk, however when NRA was performed on the back side of the samples, no deuterium was detected. Accordingly, PAS performed on the back side of the samples should give an indication of what heating does to a sample barring the effects of deuterium retention. This can be examined in Figure 1(b). Assuming that deuterium did not permeate $\sim 142 \mu \mathrm{m}$ (sample thickness minus probing depth of Na-22 positrons) to the back side of the sample, the increased S-parameter in Y107 suggests that the quantity or size of defects increases as the sample is heated to $900^{\circ} \mathrm{C}$ during TDS. The monovacancies formed during HFIR irradiation would have evolved/clustered little during D implantation at $200^{\circ} \mathrm{C}$, but heating to $900^{\circ} \mathrm{C}$ during TDS would result in clustering of vacancies, thus increasing the S-parameter. The fact that $\Delta S_{\text {front }}>\Delta S_{\text {back }}$ can be attributed to deuterium desorption from the front side of the $200^{\circ} \mathrm{C}$ sample. Now consider sample Y112. HFIR neutron irradiation produced primarily 
monovacancies, but then $\mathrm{D}$ implantation occurred at $500 \mathrm{C}$ (total of 4 hours). At this temperature void migration and clustering began. Then, since clusters would have already been present, heating to $900^{\circ} \mathrm{C}$ during TDS would drive the clusters to the surface, thus reducing the S-parameter. In semiconductors, Brusa, et al. found that the S-parameter has multiple inflection points during isothermal heating at $250^{\circ} \mathrm{C}$ for He-implanted Si [16]. Initially the S-parameter decreased, but then began to increase as the annealing time lengthened (see Fig. 6 in Ref. [16]). Likewise, it is reasonable that the $\sim 2$ hour difference in high temperature ion implantation for sample $\mathrm{Y} 112$ at $500^{\circ} \mathrm{C}$ and $\mathrm{Y} 107$ at $200^{\circ} \mathrm{C}$ is what causes Y112 to have a much lower S-parameter after TDS. Again, $\Delta S_{\text {front }}>\Delta S_{\text {back }}$ (where $\Delta S_{\text {back }}=S_{\text {Post-TDS }}-S_{\text {Pre-TDS }}$ is negative) can be attributed to deuterium desorption from the front side of the $500^{\circ} \mathrm{C}$ sample.

Positrons become trapped in voids because the positive charge of nuclei cores of the void 'shell' induce a repulsive force on the positron. Impurities decorating a void increase the positive repulsion of the void and increase the likelihood that the positron will scatter out of the void. Consequently, desorption of deuterium from voids leads to enhanced positron trapping (larger S-parameter). However, since heating to $900^{\circ} \mathrm{C}$ can potentially reduce the net defect concentration, TDS should be performed without reaching microstructural recovery stages in order to test and distinguish these phenomena (e.g., isochronal heating).

Bulk behavior of the sample is interrogated using Ge-68. In Figure 1 (c-d) the Ge-68 Sparameter is consistently higher after TDS, and the S-parameter for the $200^{\circ} \mathrm{C}$ sample is higher than the $500^{\circ} \mathrm{C}$ sample after TDS. Considering that TDS results from Figure 2 show that more deuterium was retained in the $500^{\circ} \mathrm{C}$ sample, this indicates that the source of the larger S-parameter in Y107 must be a consequence of microstructural recovery. Presently, it is unclear why the S-parameter from the back-side of Y107 (d) is higher than that of the front side (c). 


\subsection{Normalized annihilation spectrum}

Changes in the annihilation spectra from sample to sample are subtle but can be exploited

by normalizing a spectrum to that of a reference spectrum. In Figure $3,{ }^{22} \mathrm{Na}$ front-side annihilation spectra from Y107 and Y112 samples were normalized by the 0.3 dpa reference sample (Y111). Each of the three samples had $0.3 \mathrm{dpa}$ from neutron irradiation, so the changing variable in each figure is the deuterium implantation temperature. The line at $y=$ 1.0 represents the reference sample. As the normalized spectra approach this line, it reveals that the sample defects/microstructure are similar to the reference sample. Likewise, large deviations from 1.0 means that the defects/microstructure differ greatly from the reference sample. The variance at the fringes becomes sporadic due to the low counts and high background from using a single detector setup.

Prior to TDS, Figure $3(\mathrm{a})$, the normalized spectrum for the $200^{\circ} \mathrm{C}$ sample (Y107/Y111) appears broad and quite flat. Qualitatively, this means that the defects in Y107 are very similar to those found in Y111. This compares well to the S-parameters shown in Figure 1(a). In contrast, the sharper peak observed in the $500^{\circ} \mathrm{C}$ sample (Y112/Y111) in Figure 3(a) shows that the sample has fewer high-momentum annihilation electrons than the reference sample and consequently a higher defect concentration. After TDS (b), the profiles for the two samples are nearly identical, but both deviate significantly from unity. The narrow peaks indicate that the defect concentration is higher than that of the reference sample. These results are significant because they show that deuterium implantation at $200^{\circ} \mathrm{C}$ does not significantly alter the defect concentration with respect to the reference 0.3 dpa sample. Furthermore, TDS modifies both samples to have similar normalized spectra (i.e., defect concentrations) regardless of their previous history.

\section{Conclusions}


DB-PAS has been used to qualitatively investigate retention and defect concentration in neutron damaged tungsten before and after TDS. The differences in the $200^{\circ} \mathrm{C}$ and $500^{\circ} \mathrm{C}$ samples before and after TDS reveal two TDS-induced phenomena that affect the Sparameter. First, desorption of deuterium consistently results in a higher-S-parameter. Second, microstructural defect recovery can increase or decrease the S-parameter. In the $200^{\circ} \mathrm{C}$ sample microstructural recovery increased the S-parameter after TDS, but in the $500^{\circ} \mathrm{C}$ sample TDS resulted in a decreased S-parameter. This is suspected to be affected by the length of time each sample was held above stage III recovery temperatures $\left(>350-400^{\circ} \mathrm{C}\right)$, as void size and number density change with respect to time. Distinguishing these phenomena will be the focus of future work. Normalizing the raw annihilation spectra to a reference sample reveals that prior to TDS the $200^{\circ} \mathrm{C}$ sample has a qualitatively similar defect profile as the reference sample, but after TDS the profile suggests more defects than the reference sample.

\section{Acknowledgements}

We would like to thank M. Drigert for help in PAS data collection. This work was prepared for the US Department of Energy, Office of Fusion Energy Sciences, under the DOE Idaho Field Office contract number DE-AC07-05ID14517. Samples for this work came from the collaborative Japan/USA TITAN project. 


\section{References}

[1] W.R. Wampler, R.P. Doerner, Nuclear Fusion 49 (2009) 115023.

[2] Y. Oya, M. Shimada, M. Kobayashi, T. Oda, M. Hara, H. Watanabe, Y. Hatano, P. Calderoni, K. Okuno, Phys. Scr. T145 (2011) 014050.

[3] M. Shimada, Y. Hatano, Y. Oya, T. Oda, M. Hara, G. Cao, M. Kobayashi, M. Sokolov, H. Watanabe, B. Tyburska-Püschel, Y. Ueda, P. Calderoni, K. Okuno, Fusion Engineering and Design 87 (2012) 1166.

[4] M. Haaks, T.E.M. Staab, K. Saarinen, K. Maier, Phys. Status Solidi (C) 202 (2005) R38.

[5] C.N. Taylor, M. Shimada, B.J. Merrill, M.W. Drigert, D.W. Akers, Y. Hatano, Phys. Scr. T159 (2014) 014055.

[6] Standard Practice for Characterizing Neutron Exposures in Iron and Low Alloy Steels in Terms of Displacements Per Atom (DPA), E 706(ID), ASTM International, n.d.

[7] L.R. Greenwood, F.A. Garner, J. Nucl. Mater. 212 (1994) 635.

[8] M. Mayer, IPP Technical Report 9/113 (1997) (http:.

[9] M. Shimada, G. Cao, T. Otsuka, M. Hara, M. Kobayashi, Y. Oya, Y. Hatano, Nuclear Fusion submitted (2014).

[10] R.W. Siegel, Ann. Rev. Mater. Sci. 10 (1980) 393.

[11] F. Tuomisto, I. Makkonen, Rev. Mod. Phys. 85 (2013) 1583.

[12] M. Mourino, H. Löbl, R. Paulin, Physics Letters A 71 (1979) 106.

[13] P.M.G. Nambissan, P. Sen, J. Nucl. Mater. 71 (1989) 1165.

[14] V.S. Subrahmanyam, P. Nambissan, P. Sen, Solid State Communications 89 (1994) 523.

[15] H. Schultz, in:, H. Ullmaier (Ed.), Atomic Defects in Metals, Springer Berlin Heidelberg, 1991, pp. 179-190.

[16] R.S. Brusa, G.P. Karwasz, N. Tiengo, A. Zecca, F. Corni, G. Calzolari, C. Nobili, Journal of Applied Physics 85 (1999) 2390. 


\section{Figure Captions}

Figure 1. S-parameters of 0.3 dpa neutron irradiated tungsten samples before and after TDS. The reference 0.3 dpa sample did not undergo D implantation nor TDS. The pre-TDS data come from Ref. [5].

Figure 2. Deuterium retention in the two samples was measured by TDS and NRA. NRA detects deuterium from the first few microns from the surface, whereas TDS detects deuterium from the sample entirety.

Figure 3. Normalizing the ${ }^{22} \mathrm{Na}$ front-side $511 \mathrm{keV}$ annihilation peak to a reference peak (Y111) shows how a sample differs from the reference sample (Y111). Y111 was irradiated in HFIR to 0.3 dpa neutron without further modification. Y107 (solid line) and Y112 (dashed line) had deuterium implanted at 200 and $500^{\circ} \mathrm{C}$, respectively. If sample defects/concentration are identical to the reference sample, the normalized spectrum equals 1 . (a) shows the preTDS normalization, and (b) shows the post-TDS normalization. 


\section{Figures}

Figure 1

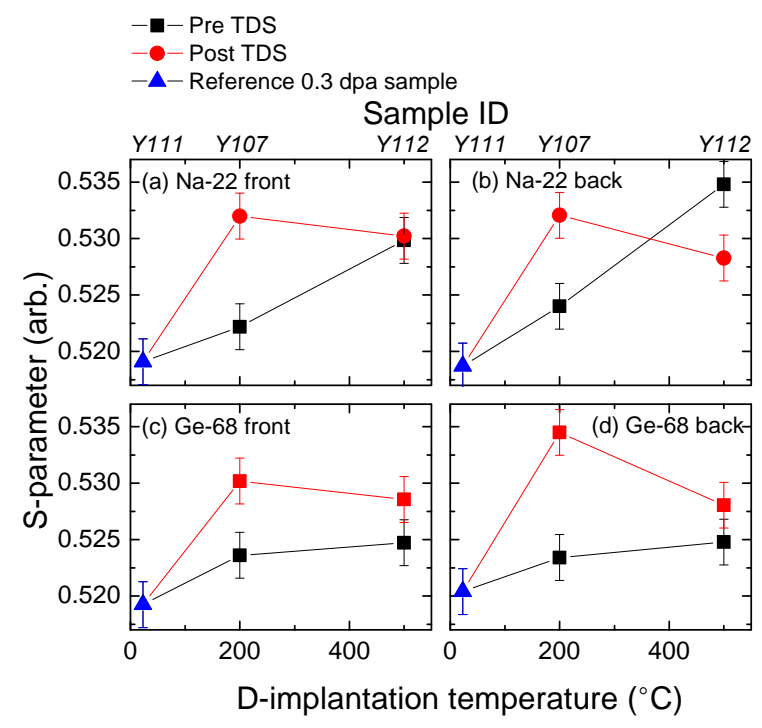


Figure 2

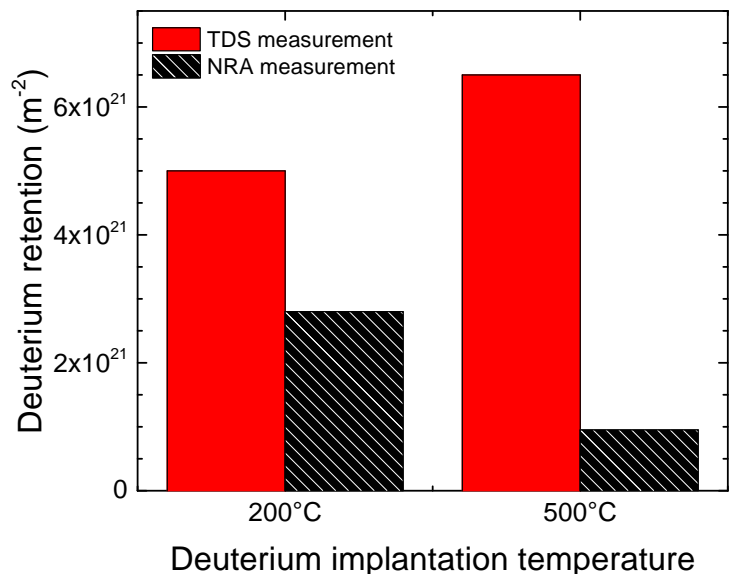


Figure 3

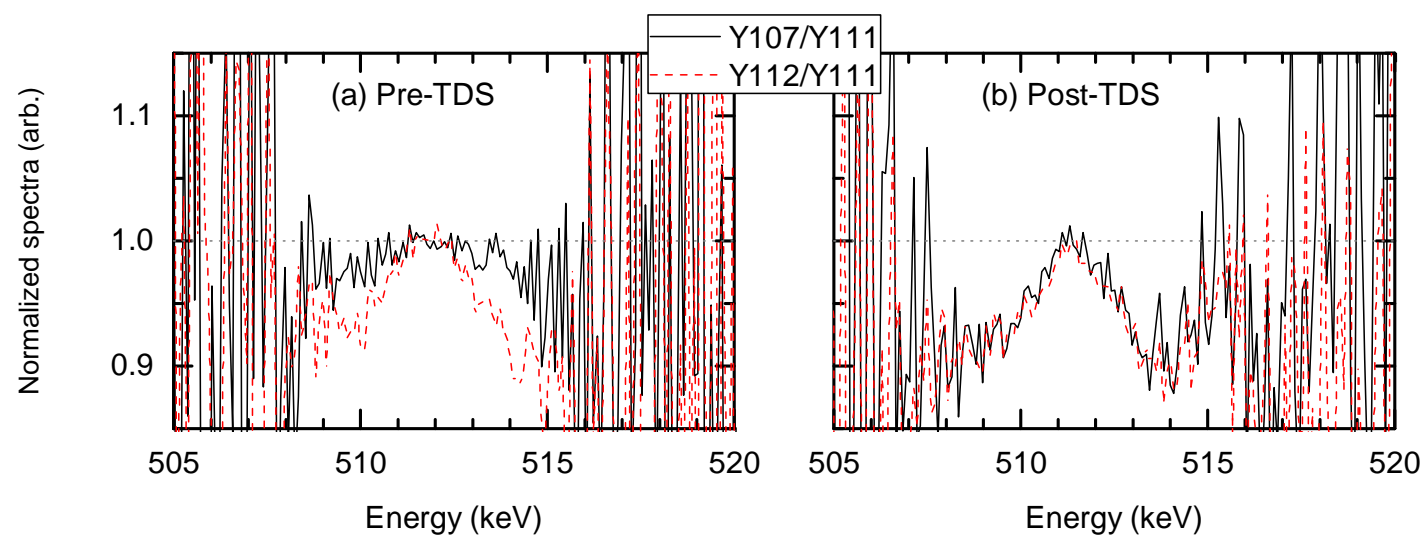

